\title{
Inhibiting two cellular mutant epidermal growth factor receptor tyrosine kinases by addressing computationally assessed crystal ligand pockets
}

\author{
Jia-Hau Lee ${ }^{1,2}$, Wei-Chen Lin ${ }^{1}$, Tsung-Kai Wen ${ }^{3}$, Chihuei Wang**,1 \& Ying-Ting Lin*,1 \\ ${ }^{1}$ Department of Biotechnology, College of Life Science, Kaohsiung Medical University, Kaohsiung, Taiwan \\ ${ }^{2}$ Graduate Institute of Natural Products, College of Pharmacy, Kaohsiung Medical University, Kaohsiung, Taiwan \\ ${ }^{3}$ School of Post-Baccalaureate Chinese Medicine, Tzu Chi University, Hualien 97004, Taiwan \\ *Author for correspondence: Tel.: +886 7322 1514; Fax: +886 7312 5339; ytlin@kmu.edu.tw \\ **Author for correspondence: chwang@kmu.edu.tw
}

Aim: Blocking receptor tyrosine kinases is a useful strategy for inhibiting the overexpression of EGFR. However, the quality of crystal pocket is an essential issue for virtually identifying new leads for surviving resistance cancer cells. Results: With the examinating crystal pocket quality by the self-docking rootmean-square deviation (RMSD) calculation, we used the two best kinase pockets of mutant EGFR kinases, T790M/L858R and G719S, for virtual screening. After sorting all the docking poses of the 57,177 library compounds by consensus scores, three evidently blocked cellular EGFR phosphorylation in the H1975 and SW48 cell lines. Conclusion: The computationally assessed qualities of crystal pockets of crystal EGFR kinases can help identify new cellular active and target-specific ligands rapidly and at low cost.

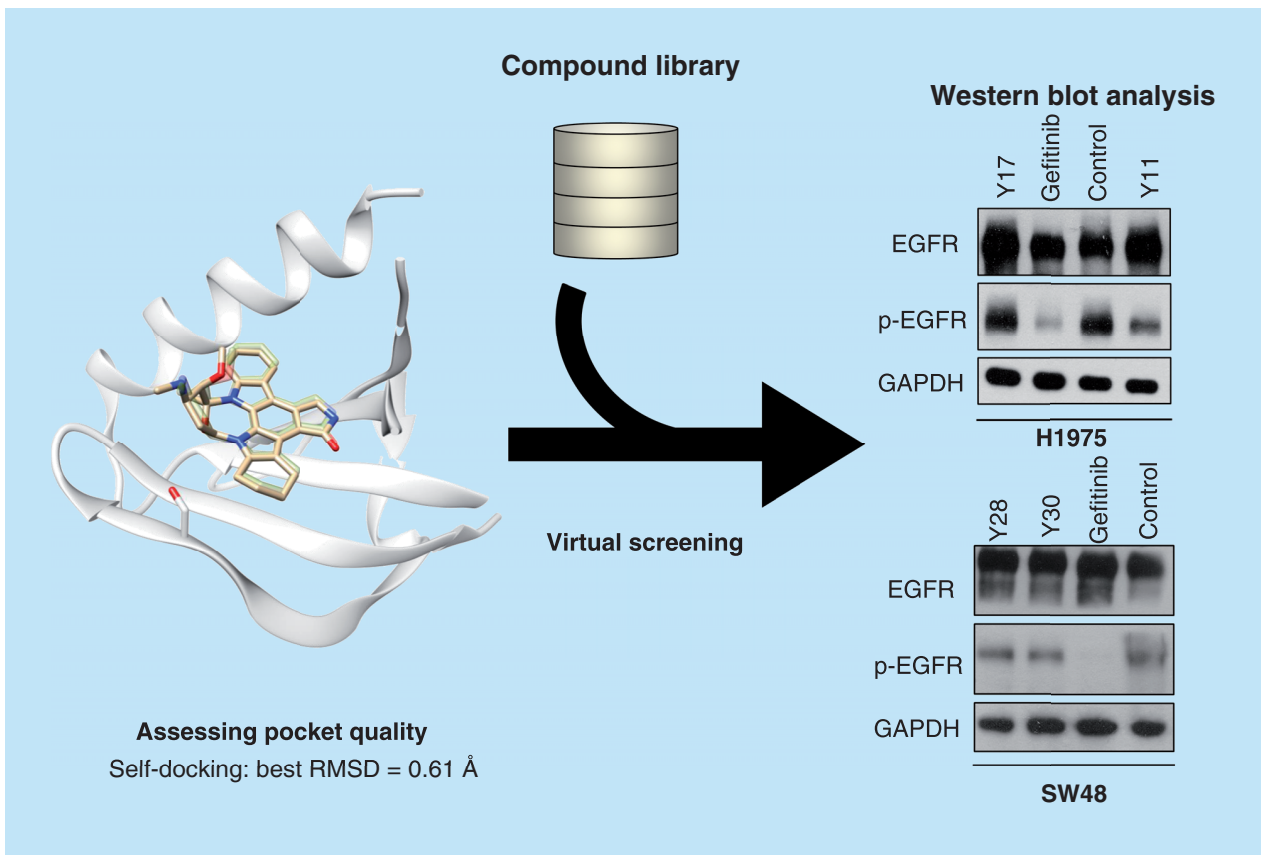

First draft submitted: 6 November 2018; Accepted for publication: 22 January 2019; Published online: 6 February 2019

Keywords: cellular bioactivity • ligand self-docking $\bullet$ NSCLC • quality crystal pocket • RMSD • RTK • structure-based virtual screening $\bullet$ TKI 
There is an urgent need to discover and develop new potent anti-non-small cell lung cancer (NSCLC) medications for both primary tumors and tumor with acquired resistance [1-3]. Many efforts aim to inhibit overexpression of EGFR [4-7]. Abnormal high activation of receptor tyrosine kinases (RTKs) is involved in carcinogenesis and tumor progression (pro-proliferative and cell survival) $[2,3,8,9]$. In 2003, the first tyrosine kinase inhibitor (TKI), Gefitinib [10] (Iressa) (Figure 1A) was approved by the US FDA. TKIs reversibly compete with ATP binding at the tyrosine kinase domain (TK) of EGFR. Unfortunately, the inefficiency of EGFR-TKIs (primary resistance) was soon discovered and cancer cells soon developed drug resistance (acquired resistance). EGFR-RTK mutations T790M, L858R and G719S are some of the common alterations [2,11]. Though next-generation EGFR-TKIs (e.g., afatinib and dacomitinib) have been developed, acquired resistance remains a challenging issue for TKI therapies $[1,3]$.

To address EGFR drug resistance, virtual screening based on the crystal structures of EGFR-TK complexed with inhibitors [12-14] provides an efficient way to identify potential therapeutic combinations. Several virtual screening approaches using molecular docking for wild-type or mutant EGFR-TKI followed by experimental confirmation have been reported in recent years [15-20]. To improve the identification of potential hits by virtual screening, kinase-focused libraries [17], natural product databases [20] and homology binding pockets [19] have been constructed beforehand for screening. Subsequent simulation of molecular dynamics [17] and hit optimization [17,19] have also improved the success rate in searching for potent inhibitors, either altering the coordinates of experimental crystal ligand pockets or conducting further structural modifications to screened hits.

To further advance this technology, we shifted our attention to the quality of experimental x-ray native binding sites (NBS). We assess the quality of crystal pocket by the root-mean-square deviation (RMSD) calculation [21] of the binding inhibitor by self-docking. When crystal EGFR kinases are complexed with inhibitors, if a kinase inhibitor regains its original crystal pose after self-docking, one can regard such ligands as being in a good binding site. This can be further chosen as a target binding site for virtual screening. In this report, we present an ultra-efficient docking-based virtual screening against Maybridge (57,177 compounds), a common and relatively small-sized chemical library, [22] for new TKIs by using LigandFit, [23] a widely used docking program [22] with a common scoring technique, consensus scoring [24-28]. Instead of an in vitro enzyme assay, we wanted to see how close a hit from this virtual screening system would be to a lead [29,30]. Therefore, we carried out cell-based assays for the screened hits. By assessing pocket quality, out of the top 20 screened ligands we found four hits that can inhibit cell proliferation (ATP consumption assay) and block cellular EGFR phosphorylation (western blotting) in the corresponding cancer cells of T790M/L858R and G719S mutants without further structural modification. In the end, the two self-docking proved binding sites with best docking poses of three potent hits are used for the examination of their key interactions. The information of binding modes can facilitate possible lead optimization. Thus, with a minimum budget of purchasing library compounds, any lab with a need for new hits against the rapidly mutated genes can efficiently screen bioactive ligands virtually through crystal ligand pocket quality. Accelerating the process of identifying diverse potent hits at very low cost may also be valuable for early drug discovery.

\section{Experimental methods Molecular modeling \& RMSD}

We performed all molecular docking experiments (including ligand self-dock and virtual screening) on an Asus personal computer with an Intel Core i7 $4.00 \mathrm{GHz}$ processor, running Windows 7 using ChemBioOffice 2010 [31] and Discovery Studio 2.5 (DS) [32]. We retrieved 79 receptor structures of human EGFR kinases co-crystallized with ligands from the Protein data bank (PDB; www.rcsb.org/pdb/index.html) [12]. We optimized each ligand within ChemBio3D of ChemBioOffice using the MMFF94 forcefield until the structure's energy reached the RMSD gradient of $0.05 \mathrm{kcal} \mathrm{mol}^{-1} \AA^{-1}$ [33]. The automatically set partial charges and the optimized structures were saved in MOL files. We carried out the self-docking strategy with a widely used docking program [22], LigandFit, using the co-crystal ligand to define the binding site which expands the occupied space of the crystal ligand by setting the ligand hydrogen radius to $2.0 \AA$ and heavy ligand atom to $2.5 \AA$ (LigandFit default). The crystal ligand was manually removed from the active site before the docking experiment. DockScore (a built-in scoring function) was used to measure the interaction energy between ligand pose and receptor. A higher score indicates stronger binding affinity. 
(A)<smiles>COc1cc2c(cc1OCCCN1CCOCC1)C1C=CC2=NC=NC(Nc2ccc(F)c(Cl)c2)=C1</smiles>

Gefitinib

(B)<smiles>O=C(Nc1cccc(Oc2ccc(Nc3ncnc4ccn(CCOCCO)c34)cc2Cl)c1)NC1CCCCC1</smiles>

3W2R ligand<smiles>Cc1nn(-c2ccccc2)nc1C(=O)Nc1nc(-c2ccc(F)c(F)c2)cs1</smiles>

Љ

(C)

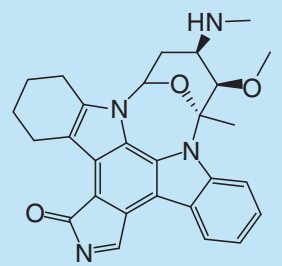

2ITQ ligand<smiles>Cc1ccc(-c2cc(-c3ccco3)c3c(n2)C(N)C(C(=O)NNC(=S)Nc2ccccc2)O3)cc1</smiles>

Y23

Y26<smiles>FC(F)(F)c1cc(NC(=S)N/N=C/C2c3ccccc3NC2c2ccc(Cl)cc2)cc(C(F)(F)F)c1</smiles><smiles>FC(F)(F)c1cccc(NC(=S)Nc2cccc(NC(=S)Nc3cccc(C(F)(F)F)c3)n2)c1</smiles>

Y28

Y30

Figure 1. Chemical structures. (A) Gefitinib. (B) Compounds $Y 11$ and $Y 17$, screened by $3 W 2 R$ ligand pocket, have significant different structures than the parent ligand. (C) Compounds Y23, Y26, Y28 and Y30, screened by 2ITQ ligand pocket, also have greatly different structures than the parent compounds. Scaffold hopping is observed in these two sets of compounds. 


\section{Virtual screening \& consensus scoring}

We used the same hardware and software for the equal base docking methods. LigandFit [23] is a molecular docking method aiming for accurately docking ligands into protein binding sites and can be used in high-throughput screening. A cavity detection algorithm is used for candidate active site regions. A Monte Carlo shape-based method has significant features for matching a small docking molecular conformation into the shape of active site. The optimization of ligand binding conformation is through a grid-based method to obtain the proteinligand interaction energies. Two types of energy functions, a soft electrostatic potential and a soft van der Waals potential, and a scheme of nonlinear interpolation were further considered and implemented to reduce errors from grid interpolation by the developers [23]. The optimal docking pocket is described above. Crystal protein residues around binding sites were not allowed to move. All crystal waters were also removed. For a single library compound, we set the maximum 100 poses able to be preserved after molecular docking. Performance was measured by 11 scoring functions, LigScore1 [23], LigScore2 [23], DockScore [23], PMF [34], PMF04 [35], Ludi1 [36], Ludi2 [36,37], Ludi3 [38], PLP1 [39,40], PLP2 [39,40] and Jain [41]. We used multiple consensus scoring criteria. If a pose's score in a scoring function was within the top $1 \%$ of total library docking poses, by definition the pose gained 1 consensus point of $1 \%$. Similarly, if within the top 3 or $5 \%$, the pose then gained 1 consensus point of 3 or $5 \%$. We chose Maybridge Screening Collection as our screening library. The last examination of the binding modes of three potent hits employed the best poses of three hits and the self-docking approved crystal pockets of mutant EGFRs, 2ITQ and $3 \mathrm{~W} 2 \mathrm{R}$. The hydrogen bond, $\pi-\pi$ interaction and $\pi$-cation interaction were checked by the distance criteria of different binding types by using DS2.5 as shown in the result and discussion section.

\section{Cell viability assay}

Cell viability was determined using the CellTiter ${ }^{\circledR}$ Glo luminescent cell viability kit from Promega Corporation (WI, USA) according to the manufacturer's instructions. Two cancer cell lines, NCIH1975 [H-1975, H1975] (ATCC ${ }^{\circledR}$ CRL5908 ${ }^{\mathrm{TM}}$ ) and SW48 [SW-48] (ATCC CCL-231 ${ }^{\mathrm{TM}}$ ), were seeded in 96-well plates and incubated for $24 \mathrm{~h}$. Then cells were treated with test ligands for 1 and 3 days $(20 \mu \mathrm{M})$. Lastly, $25 \mu \mathrm{l}$ of the reagent was added directly to each well and mixed. ATP was spectrophotometrically quantified at the $560 \mathrm{~nm}$ wavelength by luminometry. For calculating the $\mathrm{IC}_{50}$ of test ligands, the two cancer cell lines were exposed to various ligand concentrations $(0.2,0.4,0.8,1.6,3.2,6.4,12.5,25,50$ and $100 \mu \mathrm{M})$. Cancer cells were seeded and treated with test ligands as described above. ATP was then quantified and the $\mathrm{IC}_{50} \mathrm{~s}$ were calculated for each test ligand using GraphPad.

\section{Antibodies}

The following antibodies were used for western blot: EGFR (\#4267; dilution, 1:1000) phospho-EGFR Y1068 (\#2234; dilution, 1:1000), phospho-EGFR Y1068 (\#3777; dilution, 1:1000); secondary antibodies: anti-rabbit IgG, HRP-linked antibody (\#7074; dilution, 1:10,000) and anti-mouse IgG, HRP-linked antibody (\#7076; dilution, 1:10,000) from Cell Signaling (Cell Signaling Technology, Inc., MA, USA).

\section{Western blot}

Whole cell protein extracts were lysed in RIPA buffer $(25 \mathrm{mM}$ Tris-HCl pH7.6, $150 \mathrm{mM} \mathrm{NaCl}, 1 \%$ NP40 $1 \mathrm{mM}$ DTT, $0.1 \%$ NP-40, $1 \%$ sodium deoxycholate, $0.1 \%$ SDS) containing proteinase and phosphatase inhibitors by sonication. Protein concentration was determined by a Pierce ${ }^{\mathrm{TM}}$ BCA Protein Assay Kit (Thermo Fisher Scientific, MA, USA). Equal amounts of protein $(60 \mu \mathrm{g})$ were resolved by using $8 \%$ SDS-PAGE (for EGFR and p-EGFR). The total proteins were transferred to a nitrocellulose membrane after electrophoresis. The transferred membranes at room temperature were blocked in $5 \%(\mathrm{w} / \mathrm{v})$ nonfat dry milk with $0.1 \%(\mathrm{v} / \mathrm{v})$ Tween 20 for $1 \mathrm{~h}$ and probed at $4{ }^{\circ} \mathrm{C}$ overnight for the first antibody, incubated with a secondary antibody for $1 \mathrm{~h}$, bound with horseradish peroxidase and visualized by photo film development using ECL (Merck Millipore, Darmstadt, Germany).

\section{Statistical analysis}

All statistical results in figures and tables were calculated by at least four independent experiments in GraphPad Prism version 6 and are expressed as means \pm standard deviation. One-way ANOVA was performed for statistical comparison and $\mathrm{p}<0.05$ was considered significant. 


\section{Results \& discussion}

\section{Ligand self-docking, RMSD \& quality docking pockets}

Ligand self-docking is one of the significant metrics originally designed to evaluating docking methods [21] by correctly regenerating the crystal ligand pose, in which the spatial RMSD is calculated between the docked pose and crystal pose as follows:

$$
\operatorname{RMSD}=\sqrt{\frac{\sum_{i=1}^{n}\left(d x_{i}^{2}+d y_{i}^{2}+d z_{i}^{2}\right)}{n}}
$$

where $\mathrm{n}$ is the number of heavy atoms in the comparison and $d x_{i}, d y_{i}$ and $d z_{i}$ are the deviations between the ligand-docked pose and crystal pose on Cartesian coordinate $i$.

By checking the values of RMSD, researchers can determine that receptor-based molecular docking virtual screenings are correct as expected. This spatial value can account for the accuracy of molecular docking virtual screenings, such as the docking algorithm strategy, [42-45] the accuracy of scoring functions, [28,43,46-48] or even the challenge of pose prediction (Grand Challenge 2) [49,50]. Conventionally a low RMSD, usually less than $2 \AA$, indicates the correctness of the docking method [51]. In assessing RMSD, we shifted the emphasis from judging the methodology, algorithm or screening process to assessing the quality of the $\mathrm{x}$-ray ligand pocket. Leveraging ligand self-docking to assess the quality of its native binding pocket by assessing the binding pocket is as follows. First, in order to regenerate the native poses among all the existing crystal ligand-complexed binding sites, we remove the ligands from their original crystal pockets. Second, the ligands are afterward randomly translated, reoriented, thermodynamically disturbed (molecular dynamics, MM2) [52] and conformationally optimized (molecular mechanics, MMFF94) [53]. We then dock the ligands back into the native apo crystal pockets and calculate the RMSDs between the docked pose and crystal pose. For instance (Figure 2A \& B), the average RMSDs of 2ITQ and 3W2R ligands are 0.61 and $0.62 \AA$, respectively, using the three different force field energy grids, Dreiding, [54] CFF, [55-58] and PLP1 $[59,60]$. Note that an RMSD closer to zero indicates that the docked pose (semi-transparent) is spatially more similar to the native ligand pose.

In pursuit of an ultra-efficient receptor-based virtual screening for EGFR by purchasing a minimum number of library compounds. All best-docked poses of 79 human EGFR-TK ligands spatially compared with their native crystal poses gave the RMSD values after ligand self-docking are listed in Supplementary Table 1. The RMSD values ranged from 0.61 to $9.42 \AA$ and the average was $4.41 \AA$ (Figure 2C). The 2ITQ and 3W2R ligands had the lowest RMSD values. We then judged the quality of $2 \mathrm{ITQ}$ and $3 \mathrm{~W} 2 \mathrm{R}$ ligand pockets as most 'molecular dockable' [61]. We chose these two ligand binding sites for the receptor-based virtual screening of mutant EGFR-TK ligand binding. Note that 2ITQ is a crystal of mutant G719S EGFR kinase while 3W2R is a mutant T790M/L858R EGFR kinase.

Another important issue is to avoid making any unnecessary modifications to the coordinates of native ligands and pocket environments. For example, the hydrogen atoms of all crystal residues are missing due to the intrinsic limitations of protein crystallography. This means that we need to add all hydrogen atoms to the target proteins and inhibitors according to basic chemical theory by using the default values of, in this case, the CHARMM force field [62]. To avoid additional uncertainty about the crystal water oxygen atoms (with unknown orientations of hydrogen atoms attached to oxygen), we simply removed all crystal water oxygen atoms before molecular docking (even those inside the binding pocket). In such ways we can retain the unaltered structural coordinates from the experimental x-ray crystallography.

Although $\mathrm{x}$-ray crystallography can resolve protein-ligand complexes at the atomic level, the structural resolution is not a sufficient indicator for ultra-efficient receptor-based virtual screening. The average resolution of the available human EGFR kinases crystals in the PDB data bank [12] (www.rcsb.org/pdb/index.html) is $2.66 \AA$ (Supplementary table 2). Nevertheless, high crystal ligand resolution does not ensure lower RMSD values after self-docking. A 1 A deviation at a critical residue around the binding site could apparently lead to defects in docking screening. Therefore, a careful examination of the 'dockable nature' of the ligand pockets of each crystal complex with equal docking methodologies is essential for efficient docking screening. 

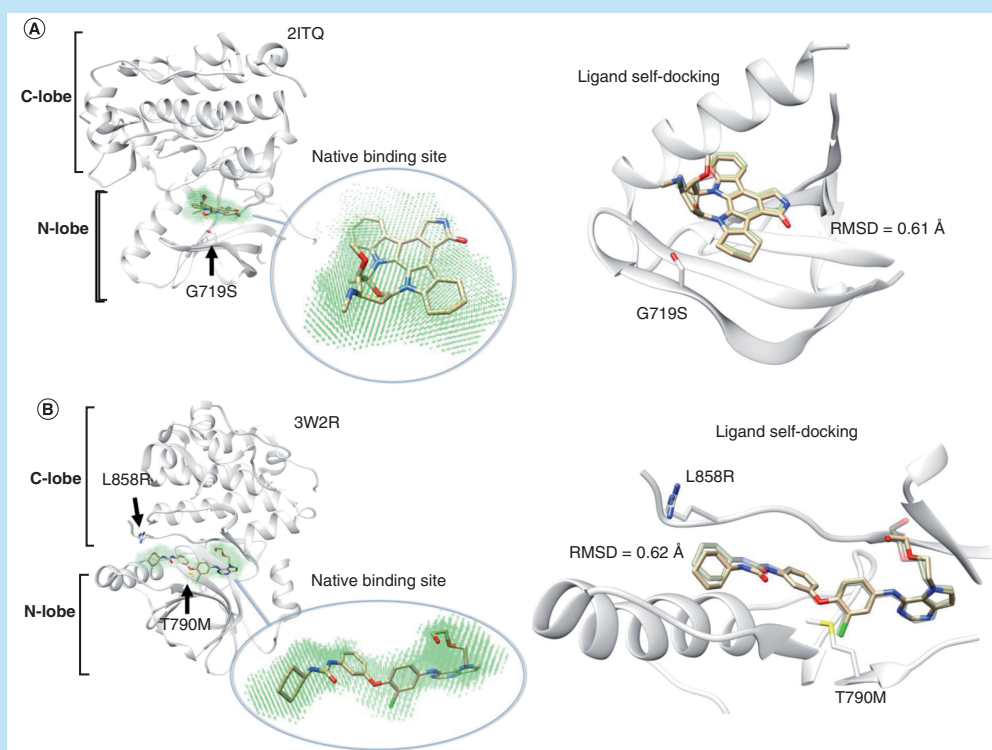

(C)
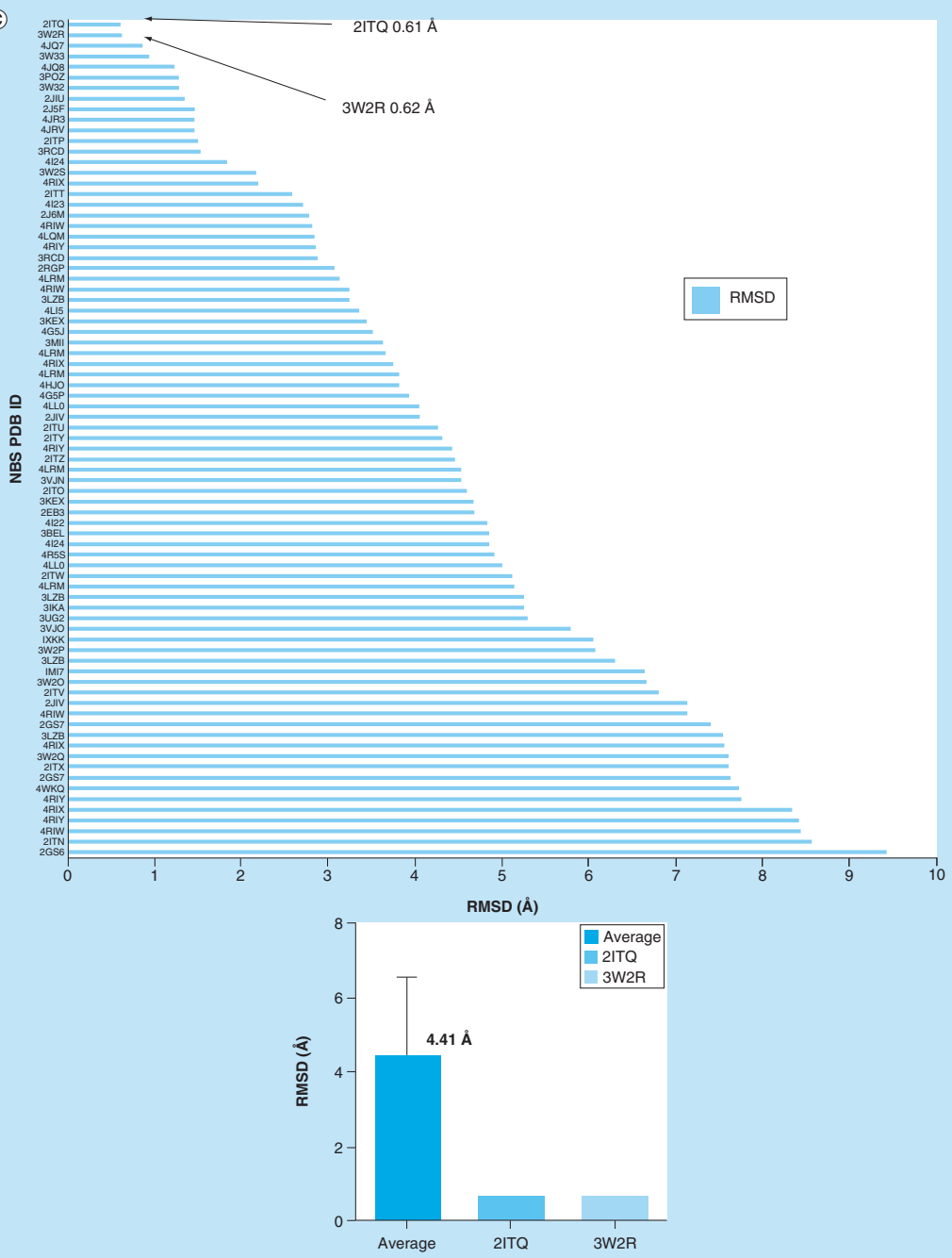

Figure 2. Ligand self-docking. (A) The average spatial RMSD, $0.61 \AA$, was calculated between the docked pose (semi-transparent) and crystal pose of 2ITQ ligand. (B) The average spatial RMSD, $0.62 \AA$, was calculated between the docked pose (semi-transparent) and crystal pose of 3W2R ligand. (C) The bar graph shows that all RMSD values of 79 docked poses of human EGFR-TK inhibitors ranged from 0.61 to $9.42 \AA$ with an average of $4.41 \AA$. All docked poses were compared spatially with their native crystal poses after ligand self-docking. The 2ITQ and 3W2R ligands have the lowest RMSD values after the self-docking.

NBS: Native binding sites; PDB: Protein data bank; RMSD: Root-mean-square deviation. 
(A)

H1975

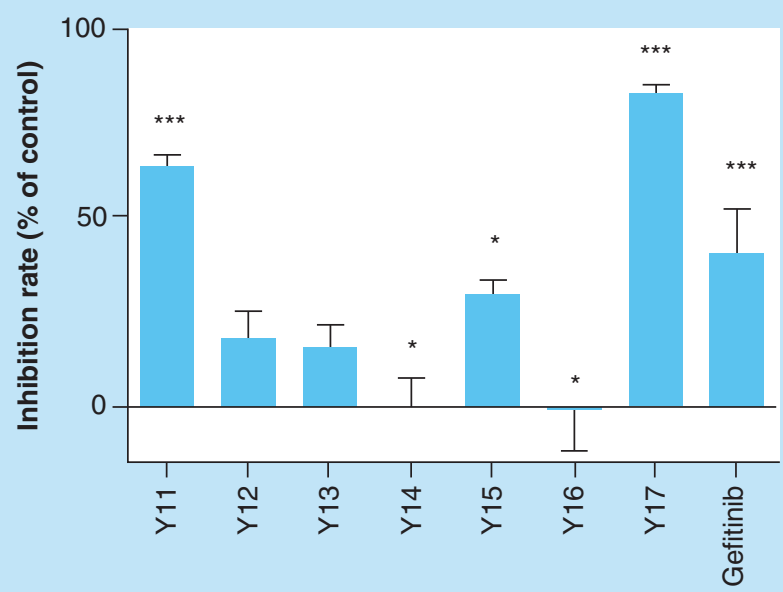

Concentration $(20 \mu \mathrm{M})$
(B)

SW48

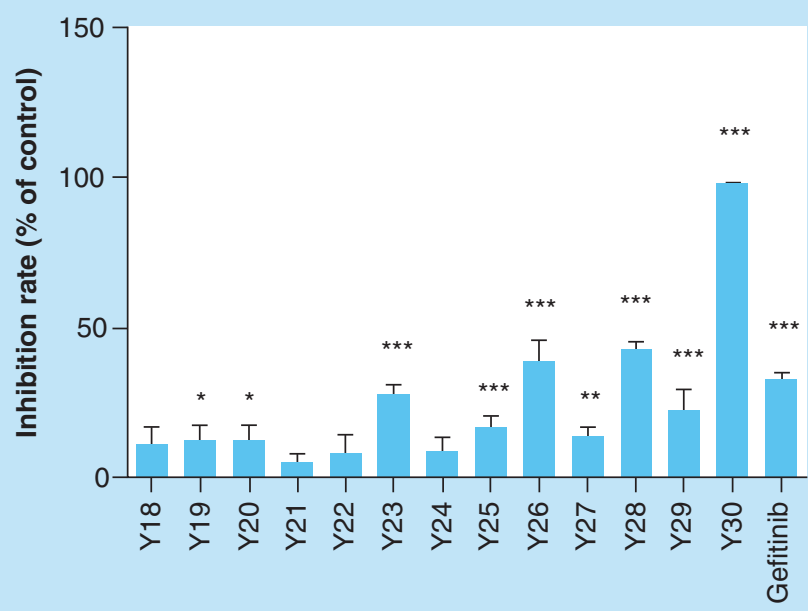

Concentration $(20 \mu \mathrm{M})$

Figure 3. The inhibition rates of screened compounds from the Maybridge library at $20 \mu \mathrm{M}$ determined by ATP consumption assays. (A) In H1795 cells, compounds Y11 and Y17 showed significant inhibition, better than Gefitinib. (B) In SW48 cells, compounds Y23, Y26, Y28 and $\mathrm{Y} 30$ showed significant inhibition, better than or comparable with Gefitinib. Data are means \pm standard deviation $(n=4)$. $* * * p<0.001, * * p<0.01,{ }^{*} p<0.05$ vs control group.

Table 1. The IC $C_{50}$ values of the selected compounds $Y 11, Y 17, Y 28, Y 30$ and gefitinib on NCI-H1975 and SW48 cells. Compound

Y11

Y17 Cell line

Gefitinib

H1975 Mean $\mathrm{IC}_{50} \pm(\mu \mathrm{M})$

Y28 H1975

$12.52 \pm 0.37$

$7.74 \pm 0.95$

Y30

H1975

$11.75 \pm 0.31$

SW48

$1.53 \pm 0.15$

Gefitinib

SW48

$8.01 \pm 0.31$

SW48

$15.92 \pm 0.99$

Relative small compound libraries, 11 scoring functions \& pose-based consensus scoring

We expect ultra-efficient receptor-based virtual screening to identify potential hits. A potent hit with more desirable properties, solubility or cell permeability can advance to the subsequent lead optimization. We chose a common screening chemical database [22] (Maybridge Screening Collection, a relatively small one with a total of 57,177 commercially available chemicals, www.maybridge.com) as our screening library. Since most scoring functions have their own preferences, [23] to eliminate scoring bias we performed consensus scoring using all 11 scoring functions in LigandFit. Each library compound can have up to 100 after-docking poses and each pose had 11 scores. If a pose's score was within the top $1 \%$ of the entire library of docking poses, by definition this pose gains 1 consensus point of $1 \%$. Similarly, if within the top 3 or $5 \%$, the pose then gains 1 consensus point of 3 or $5 \%$. An in-house Ruby script (Ruby Version 2.1.8, www.ruby-lang.org/) for the pose-based consensus scoring was used for pose-based consensus scoring. To keep the number of purchased compounds reasonable, we combined multiple consensus scorings criteria to select the final compounds. For 3W2R, we selected seven compounds, Y11-Y17, which reoccur in the two sets of top poses with 7 and 10 consensus points of 1 and 3\%. For 2ITQ, we selected 13 compounds, Y18-Y30, which repeat in the three sets of top poses with 9, 10 and 11 consensus points of 1,3 and 5\%. The selected compounds are listed in Supplementary Table 3. Using this consensus of three scoring criteria led us to exclude an extremely good compound pose with nine consensus points of $1 \%$ but which did not reach 11 consensus points of 5\%, which was below the threshold we set. Thus, we retained a few excellent compounds based on the best overall scoring functions. The structures of the identified ligands are shown in Supplementary Figure 1. We 

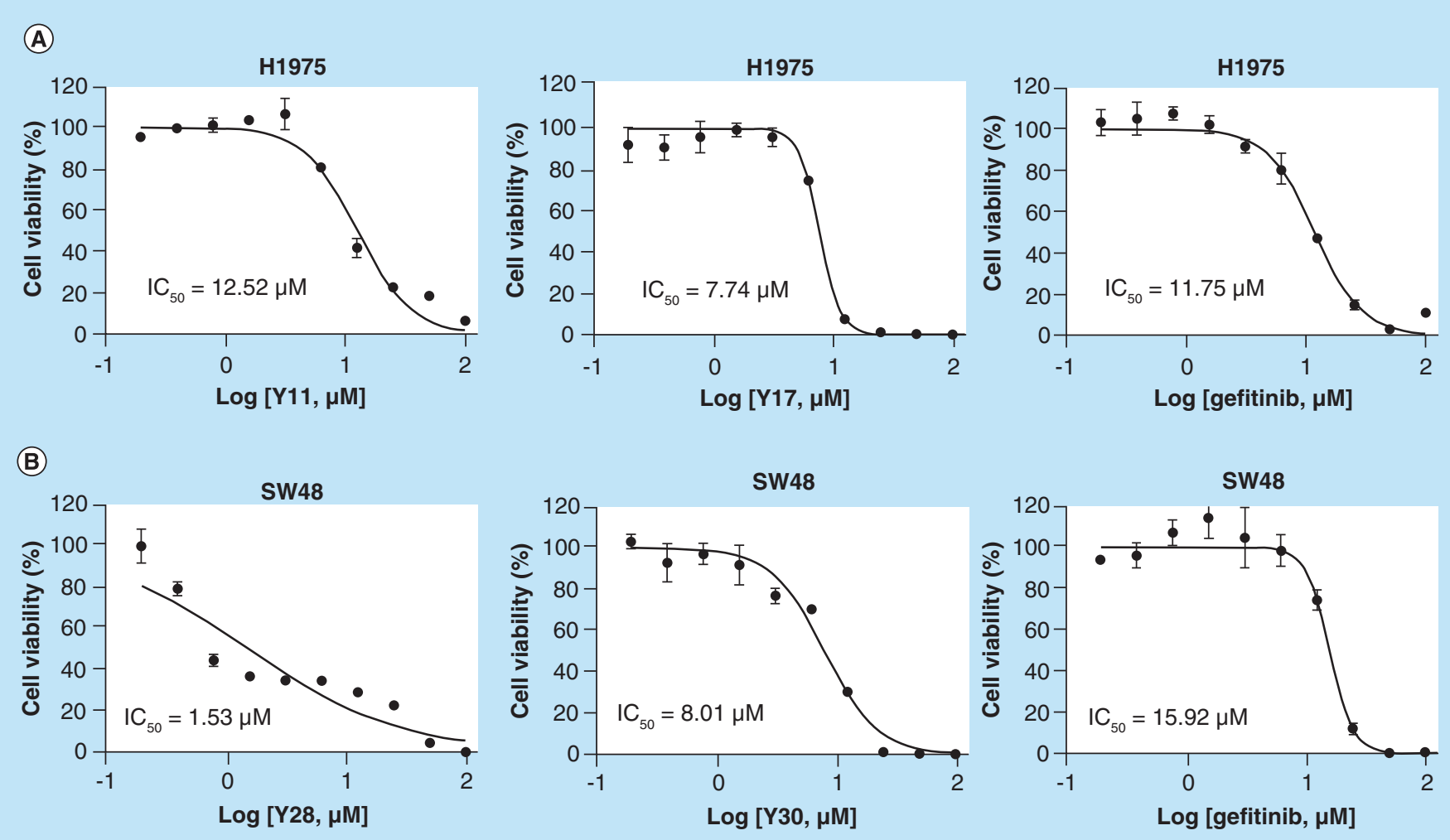

Figure 4. Dose-response curve of inhibition rate to series concentrations of the $\mathrm{Y} 11, \mathrm{Y} 17, \mathrm{Y} 28, \mathrm{Y} 30$ compounds and gefitinib on two human tumor cell lines. (A) H1975 cells and (B) SW48 cells. Cells were treated with test compounds at a series of indicated concentrations and viable cells were measured after $72 \mathrm{~h}$ of treatment. All error bars represent the mean $\pm \operatorname{standard}$ deviation $(n=4)$.

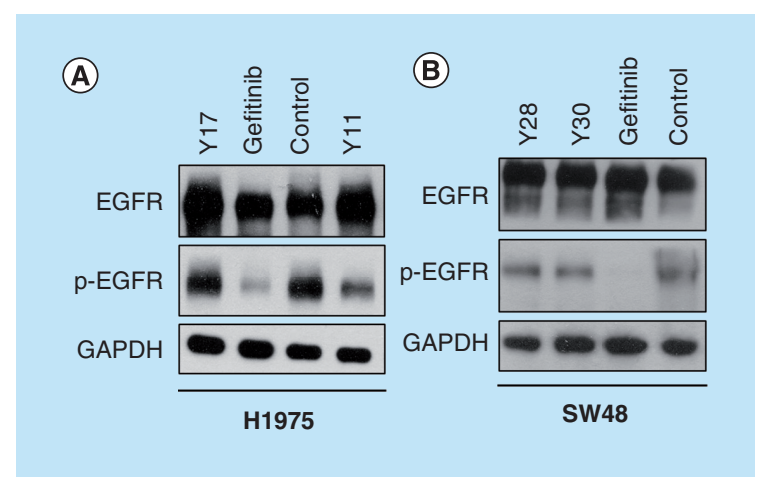

Figure 5. $\mathrm{Y} 11$ and $\mathrm{Y} 17$ decreased the expression of phosphorylated EGFR in $\mathrm{H} 1975$ cells while Y28 and Y30 decreased it in SW48 cells. (A) EGFR and phospho-EGFR expression was detected by western blot analysis in $\mathrm{H} 1975$ cells treated with Y11, Y17 and Gefitinib for $24 \mathrm{~h}$. (B) EGFR and phospho-EGFR expression was detected by western blot analysis in SW48 cells treated with Y28, Y30 and Gefitinib for $24 \mathrm{~h}$. GAPDH was measured as a loading control.

then used these two sets of compounds, Y11-Y17 (for T790M/L858R mutant EGFR-TK) and Y18-Y30 (for G719S mutant EGFR-TK), for cell viability assays and western blotting.

Cell viability assay on screened hits, $\mathrm{IC}_{50}$ \& inhibition of EGFR-TK phosphorylation

Blocking EGFR-RTK will cause cell death, especially for cancer cells with abnormal or high activation of such genes for proliferation and cell survival. Since the identified Y11-Y17 compounds screened by molecular docking interfere with the mutant T790M/L858R kinase (3W2R) and the Y18-Y30 compounds interfere with the mutant G719S kinase (2ITQ), we performed the ATP consumption assay (a cell viability assay) for all the compounds using two appropriate cell lines, H1975 and SW48. H1975 is a human lung cancer cell line expressing mutant T790M/L858R EGFR-TK. SW48 is a human colon adenocarcinoma cell line expressing mutant G719S EGFR. 


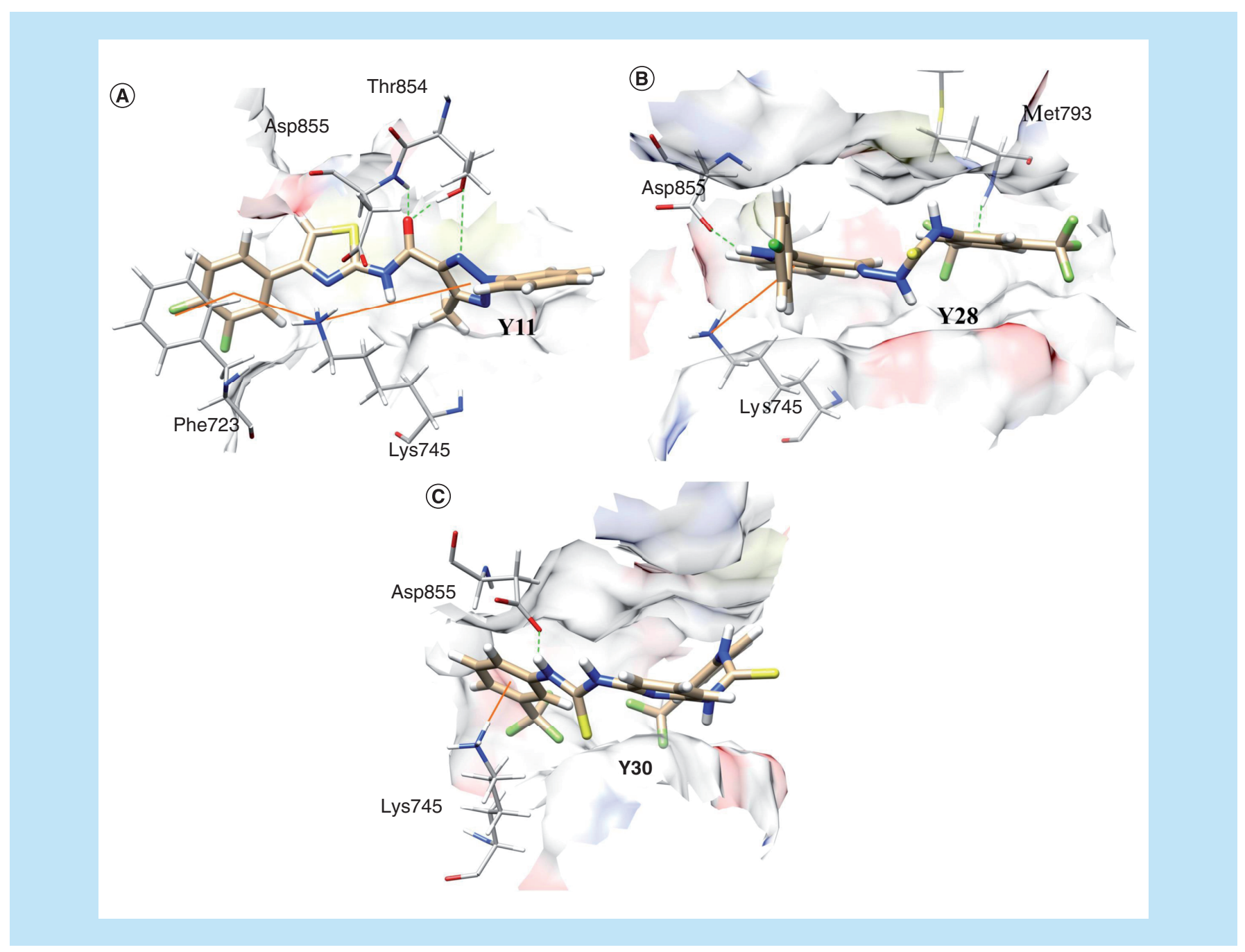

Figure 6. 3D ligand-receptor interaction diagrams for the binding site of 3W2R and 2ITQ with Y11, Y28 and Y30, respectively. (A) Binding mode of $Y 11$ in 3W2R pocket. (B) Binding mode of Y28 in 2 IYQ pocket. (C) Binding mode of Y30 in 2 IYQ pocket. The hydrogen bonds are represented in green dotted lines while $\pi$-cation interactions and $\pi$-cation interactions in orange lines. The carbon atoms of proteins are colored white. The carbon atoms of hits are colored tan.

Cell viability assays showed that two compounds, Y11 and Y17 (Figure 1B), had a significantly different core structure than the parent compound, 3W2R ligand. The two compounds significantly inhibited the proliferation of the H1975 cell line, with better results than Gefitinib (Figure 3A). Four compounds, Y23, Y26, Y28 and Y30 (Figure 1C), all had a hugely different scaffold than the parent compound, 2ITQ ligand. All four compounds significantly inhibited the SW48 cell line, with results better than or comparable with Gefitinib (Figure 3B). These two sets of compounds exhibited one important function of virtual screening, scaffold hopping [63,64]. Overall, the results of this preliminary assay had hit to test ratios of 2 to 7 and 4 to 13, showing that virtual screenings were significantly more efficient than the common high-throughput screening (HTS) ratio of hit to test, which is around 1 to 1000 [65]. We further evaluated the $\mathrm{IC}_{50}$ of Y11, Y17, Y28 and Y30 by cell viability assays for $72 \mathrm{~h}$ in two cell lines. The dose-dependent $\mathrm{IC}_{50}$ of $\mathrm{Y} 11$ and $\mathrm{Y} 17$ was 12.52 and $7.74 \mu \mathrm{M}$ for $\mathrm{H} 1975$ cells (Figure 4A) while the $\mathrm{IC}_{50}$ of $\mathrm{Y} 28$ and $\mathrm{Y} 30$ was 1.53 and $8.01 \mu \mathrm{M}$ for SW48 cells (Figure $4 \mathrm{~B}$ ). Since the $\mathrm{IC}_{50}$ values of Gefitinib showed $11.75 \mu \mathrm{M}$ for H1975 cells and $15.92 \mu \mathrm{M}$ on SW48 cells (Table 1), all these compounds showed a lower $\mathrm{IC}_{50}$ than Gefitinib. $\mathrm{IC}_{50}$ results of cell viability assays for $24 \mathrm{~h}$ were similar and are also shown for comparison (Supplementary Figures 2A \& B).

To further confirm whether the four inhibitors, Y11, Y17, Y28 and Y30, can actually inhibit cellular enzyme phosphorylation of the EGFR T790M/L858R and G719S kinases, we performed western blot analysis on these 
four compounds using the corresponding H1975 (T790M/L858R) and SW48 (G719S) cancer cells. Treating H1975 cells at $10 \mu \mathrm{M}$, Y11 effectively reduced the expression levels of phospho-EGFR. Y17 had no obvious effect. In SW48 cells, Y28 effectively reduced the expression levels of phospho-EGFR (Figure 5). The results suggested that these inhibitors can reduce EGFR phosphorylation by effectively blocking the mutant EGFR T790M/L858R and G719S kinases. The standard, gefitinib (serving as positive control), is known as an excellent binder and also can inhibit the phosphorylation of various mutant EGFR-TKs [66-71].

Our results collectively present an ultra-efficient docking-based virtual screening process considering only the ligand binding (screening hits by molecular docking) by addressing the quality of crystal ligand pockets. Without evaluating other factors in the cellular situation, we carried out two types of cell-based assays on the screened hits to examine how close our identified hits were to a lead. Out of top 20 screened ligands, three hits inhibited cell proliferation (ATP consumption assay) and blocked cellular EGFR phosphorylation (western blotting) in the T790M/L858R and G719S mutant cell lines. Assessing the quality of crystal ligand pockets shows great promise for enhancing the efficiency of molecular docking virtual screening and can be useful in the early stages of drug discovery.

\section{Prediction of the binding interactions}

The best docking poses of three most potent hits, Y11, Y28 and Y30, with highest consensus scoring were analyzed to predict their binding interactions. During the screening, Y11 was originally docked into the native apo crystal pockets of 3W2R while the other two, Y28 and Y30 were docked into that of 2ITQ. As shown in Figure 6A, Y11 had strong $\pi-\pi$ interaction with Phe 723 , two $\pi$-cation interactions with Lys 745 and hydrogen bonds with the backbone of Thr845 and Asp855. In Figure 6B, Y28 had strong $\pi$-cation interactions with Lys745 and hydrogen bonds with Met793 and Asp855. Also, in Figure 6C, Y30 formed strong $\pi$-cation interactions with Lys745 and hydrogen bond with Asp855. Three most potent hits are sharing the common features, bindings with Lys 745 and Asp855, which may shed light on the structure-activity relationship (SAR) of mutant EGFR kinase for further lead optimization.

\section{Conclusion}

Structure-based virtual screening has been used as a cost lowering strategy in early-stage drug discovery, but its efficiency regarding molecular docking can be upgraded. In this report, we demonstrated ultra-efficiently screening for new hits on two T790M/L858R and G719S mutant EGFR tyrosine kinases. We identified the new hits at very low cost and the hits also showed a shorter distance from hit to lead by rapidly revealing more lead-like properties. We suggest that the crystal ligand pocket quality plays an essential role that can be assessed by the spatial RMSD values of the redock ligand poses by an equal base molecular docking method. With the best dockable molecular pocket, a small chemical library and a widely used docking program, we identified inhibitors against mutant EGFR-TKs that did not require any further structural modification, and evaluated the direct cellular effects of these inhibitors by western blotting and cell proliferation assays. Such redock RMSD checks appear to be particularly useful for generating more hits on diverse structures in the initial phase of the drug discovery process to address rapidly mutating EGFR genes. Also, this virtual screening approach can be efficiently applied using other mutant targets and databases, which can ultimately provide potent hits with insight on the diverse novel scaffolds of TKI.

\section{Future perspective}

In the future, drug resistance is still unavoidable and there is still a huge demand to discover and develop new potent anti-NSCLC medications for both primary tumors and tumor with acquired resistance. Molecular docking is one of the most useful tools for drug discovery. In this case, from the top 20 screened ligands we efficiently found four new hits that can inhibit cell proliferation and block cellular EGFR phosphorylation in the corresponding cancer cells. Moreover, the revealed binding interactions for potent hits with different mutant EGFRs will also facilitate future drug discovery and lead optimization. We conclude that computationally assessed qualities of crystal pockets is an important checkpoint which can shorten hit-to-lead distance and also help discover structurally novel cellular active, target-specific ligands promptly and costless. 


\section{Summary points}

Root-mean-square deviation \& quality docking pockets

- Conventionally a low root-mean-square deviation (RMSD), usually less than $2 \AA$, indicates the correctness of the docking method.

- High crystal ligand resolution does not ensure lower RMSD values after self-docking.

- In all 79 native binding sites on crystal EGFR kinases, the RMSD values ranged from 0.61 to $9.42 \AA$ and the average was $4.41 \AA$. The best RMSDs of 2 ITQ and 3W2R ligands are 0.61 and $0.62 \AA$, respectively, serving as quality docking pockets.

Scoring functions \& pose-based consensus scoring

- In order to eliminate scoring bias, we performed consensus scoring using all 11 scoring functions in LigandFit with the best dockable molecular pockets.

- To keep the number of purchased compounds reasonable, we selected seven compounds for mutant T790M/L858R EGFR kinase and 13 compounds for mutant G719S EGFR kinase by using multiple consensus scorings criteria.

Cell viability assay \& inhibition of EGFR tyrosine kinase phosphorylation

- The results of cell viability assay had hit to test ratios of 2 to 7 and 4 to 13 , showing that virtual screenings were significantly more efficient than the common high-throughput screening (HTS) ratio of hit to test.

- Screened Y11, Y28 and Y30 inhibited cell proliferation (ATP consumption assay) and blocked cellular EGFR phosphorylation (western blotting) in the T790M/L858R and G719S mutant cell lines.

Supplementary data

To view the supplementary data that accompany this paper please visit the journal website at: www.futuremedicine.com/doi/full/10.2217/fmc-2018-0525

Financial \& competing interests disclosure

The authors thank the Ministry of Science and Technology of Taiwan for financial support (Grant Nos. MOST106-2113-M-037-018 and MOST107-2119-M-037-001-MY2) and also the Kaohsiung Medical University Research Foundation for funding assistance (Grant No. KMU-M106008). The authors have no other relevant affiliations or financial involvement with any organization or entity with a financial interest in or financial conflict with the subject matter or materials discussed in the manuscript apart from those disclosed.

No writing assistance was utilized in the production of this manuscript.

\section{Ethical conduct of research}

The authors state that they have obtained appropriate institutional review board approval or have followed the principles outlined in the Declaration of Helsinki for all human or animal experimental investigations. In addition, for investigations involving human subjects, informed consent has been obtained from the participants involved.

\section{Open access}

This work is licensed under the Attribution-NonCommercial-NoDerivatives 4.0 Unported License. To view a copy of this license, visit http://creativecommons.org/licenses/by-nc-nd/4.0/

\section{References}

Papers of special note have been highlighted as: $\bullet$ of interest; $\bullet \bullet$ of considerable interest

1. Tong CWS, Wu WKK, Loong HHF, Cho WCS, To KKW. Drug combination approach to overcome resistance to EGFR tyrosine kinase inhibitors in lung cancer. Cancer Lett. 405, 100-110 (2017).

2. Ke EE, Wu YL. EGFR as a pharmacological target in EGFR-mutant non-small-cell lung cancer: where do we stand now? Trends Pharmacol. Sci. 37(11), 887-903 (2016).

- Briefed the history of EGFR drug development and future perspectives.

3. Nan X, Xie C, Yu X, Liu J. EGFR-TKI as first-line treatment for patients with advanced EGFR mutation-positive non-small-cell lung cancer. Oncotarget 8(43), 75712-75726 (2017).

4. Jaramillo ML, Banville M, Collins C, Paul-Roc B, Bourget L, O’Connor-Mccourt M. Differential sensitivity of A549 non-small lung carcinoma cell responses to epidermal growth factor receptor pathway inhibitors. Cancer Biol. Ther. 7(4), 557-568 (2008).

5. Tong Y, Huang $\mathrm{C}$, Zhang J. A novel EGFR-TKI inhibitor (cAMP- $\mathrm{H}_{3} \mathrm{BO}_{3}$ complex) combined with thermal therapy is a promising strategy to improve lung cancer treatment outcomes. Oncotarget 8(34), 56327-56337 (2017). 
6. Zhong L, Yang J, Cao Z et al. Preclinical pharmacodynamic evaluation of drug candidate SKLB-178 in the treatment of non-small cell lung cancer. Oncotarget 8(8), 12843-12854 (2017).

7. Yuan JW, Qiu HY, Wang PF et al. Synthesis of caffeic acid amides bearing 2,3,4,5-tetra-hydrobenzo[b][1,4]dioxocine moieties and their biological evaluation as antitumor agents. Molecules 19(6), 7269-7286 (2014).

8. Tu HY, Ke EE, Yang JJ et al. A comprehensive review of uncommon EGFR mutations in patients with non-small cell lung cancer. Lung Cancer 114, 96-102 (2017).

9. Siegel-Lakhai WS, Beijnen JH, Schellens JH. Current knowledge and future directions of the selective epidermal growth factor receptor inhibitors erlotinib (Tarceva) and gefitinib (Iressa). Oncologist 10(8), 579-589 (2005).

10. Cohen MH, Williams GA, Sridhara R, Chen G, Pazdur R. FDA drug approval summary: gefitinib (ZD1839; Iressa) tablets. Oncologist 8(4), 303-306 (2003).

11. Singh M, Jadhav HR. Targeting non-small cell lung cancer with small-molecule EGFR tyrosine kinase inhibitors. Drug Discov. Today 23(3), 745-753 (2017).

12. Berman HM, Westbrook J, Feng Z et al. The protein data bank. Nucleic Acids Res. 28(1), 235-242 (2000).

13. Sogabe S, Kawakita Y, Igaki $S$ et al. Structure-based approach for the discovery of Pyrrolo[3,2-d]pyrimidine-based EGFR T790M/L858R mutant inhibitors. ACS Med. Chem. Lett. 4(2), 201-205 (2013).

-• Reported the information of $x$-ray mutant EGFR T790M/L858R kinase structure.

14. Yun CH, Boggon TJ, Li Y et al. Structures of lung cancer-derived EGFR mutants and inhibitor complexes: mechanism of activation and insights into differential inhibitor sensitivity. Cancer Cell 11(3), 217-227 (2007).

-• Reported the information of $x$-ray mutant EGFR G719S kinase structure.

15. Cavasotto CN, Ortiz MA, Abagyan RA, Piedrafita FJ. In silico identification of novel EGFR inhibitors with antiproliferative activity against cancer cells. Bioorg. Med. Chem. Lett. 16(7), 1969-1974 (2006).

16. Li S, Sun X, Zhao H, Tang Y, Lan M. Discovery of novel EGFR tyrosine kinase inhibitors by structure-based virtual screening. Bioorg. Med. Chem. Lett. 22(12), 4004-4009 (2012).

17. Zhao H, Dong J, Lafleur K, Nevado C, Caflisch A. Discovery of a novel chemotype of tyrosine kinase inhibitors by fragment-based docking and molecular dynamics. ACS Med. Chem. Lett. 3(10), 834-838 (2012).

18. Allen BK, Mehta S, Ember SW, Schonbrunn E, Ayad N, Schurer SC. Large-scale computational screening identifies first in class multitarget inhibitor of EGFR kinase and BRD4. Sci. Rep. 5, 16924 (2015).

19. Park H, Jung HY, Mah S, Hong S. Discovery of EGF receptor inhibitors that are selective for the d746-750/T790M/C797S mutant through structure-based de novo design. Angew. Chem. Int. Ed. Engl. 56(26), 7634-7638 (2017).

20. Zang SZ, Yang YR, Zhao SS, Li YX, Gao XY, Zhong CL. In silico insight into EGFR treatment in patients with lung carcinoma and T790M mutations. Exp. Ther. Med. 13(5), 1735-1740 (2017).

21. Fogel GB, Corne DW, Pan Y. In: Kelemen, Arpad, Abraham, Ajith, Chen, Yuehui. (Eds). Computational Intelligence in Bioinformatics. John Wiley \& Sons, NJ, USA, (2007).

22. Cheng T, Li Q, Zhou Z, Wang Y, Bryant SH. Structure-based virtual screening for drug discovery: a problem-centric review. AAPS J. 14(1), 133-141 (2012).

23. Venkatachalam CM, Jiang X, Oldfield T, Waldman M. LigandFit: a novel method for the shape-directed rapid docking of ligands to protein active sites. J. Mol. Graph. Model. 21(4), 289-307 (2003).

24. Oda A, Tsuchida K, Takakura T, Yamaotsu N, Hirono S. Comparison of consensus scoring strategies for evaluating computational models of protein-ligand complexes. J. Chem. Inf. Model. 46(1), 380-391 (2006).

25. Clark RD, Strizhev A, Leonard JM, Blake JF, Matthew JB. Consensus scoring for ligand/protein interactions. J. Mol. Graph. Model. 20(4), 281-295 (2002).

26. Charifson PS, Corkery JJ, Murcko MA, Walters WP. Consensus scoring: a method for obtaining improved hit rates from docking databases of three-dimensional structures into proteins. J. Med. Chem. 42(25), 5100-5109 (1999).

27. Wang R, Wang S. How does consensus scoring work for virtual library screening? An idealized computer experiment. J. Chem. Inf. Comput. Sci. 41(5), 1422-1426 (2001).

28. Huang SY, Grinter SZ, Zou X. Scoring functions and their evaluation methods for protein-ligand docking: recent advances and future directions. Phys. Chem. Chem. Phys. 12(40), 12899-12908 (2010).

29. Bleicher KH, Bohm HJ, Muller K, Alanine AI. Hit and lead generation: beyond high-throughput screening. Nat. Rev. Drug Discov. 2(5), 369-378 (2003).

30. Keseru GM, Makara GM. Hit discovery and hit-to-lead approaches. Drug Discov. Today 11(15-16), 741-748 (2006).

31. Kerwin SM. ChemBioOffice ultra 2010 suite. J. Am. Chem. Soc. 132(7), 2466-2467 (2010).

32. Accelrys_Software_Inc. Discovery studio modeling environment. Release 2.1 Discovery Studio Modeling Environment (2008). 
33. Halgren AT. Merck molecular force field. I. Basis, form, scope, parameterization, and performance of MMFF94. J. Comput. Chem. 17(5 \& 6), 490-519 (1995).

34. Muegge I, Martin YC. A general and fast scoring function for protein-ligand interactions: a simplified potential approach. J. Med. Chem. 42(5), 791-804 (1999).

35. Muegge I. PMF scoring revisited. J. Med. Chem. 49(20), 5895-5902 (2006).

36. Böhm HJ. On the use of LUDI to search the fine chemicals directory for ligands of proteins of known three-dimensional structure. $J$. Comput. Aided Mol. Des. 8(5), 623-632 (1994).

37. Böhm HJ. The development of a simple empirical scoring function to estimate the binding constant for a protein-ligand complex of known three-dimensional structure. J. Comput. Aided Mol. Des. 8(3), 243-256 (1994).

38. Böhm HJ. Prediction of binding constants of protein ligands: a fast method for the prioritization of hits obtained from de novo design or 3D database search programs. J. Comput. Aided Mol. Des. 12(4), 309-323 (1998).

39. Gehlhaar DK, Verkhivker GM, Rejto PA et al. Molecular recognition of the inhibitor AG-1343 by HIV-1 protease: conformationally flexible docking by evolutionary programming. Chem. Biol. 2(5), 317-324 (1995).

40. Parrill A. In: Parrill AL, Reddy MR. (Eds). Rational Drug Design: Novel Methodology and Practical Applications. Division of Computers in Chemistry, American Chemical Society. DC, USA. (1999).

41. Jain AN. Scoring noncovalent protein-ligand interactions: a continuous differentiable function tuned to compute binding affinities. J. Comput. Aided Mol. Des. 10(5), 427-440 (1996).

42. Guedes IA, De Magalhaes CS, Dardenne LE. Receptor-ligand molecular docking. Biophys. Rev. 6(1), 75-87 (2014).

43. Neves MA, Totrov M, Abagyan R. Docking and scoring with ICM: the benchmarking results and strategies for improvement. J. Comput. Aided Mol. Des. 26(6), 675-686 (2012).

44. Ashtawy HM, Mahapatra NR. Boosted neural networks scoring functions for accurate ligand docking and ranking. J. Bioinform. Comput. Biol. 16(2), 185 (2018).

45. Hoffer L, Chira C, Marcou G, Varnek A, Horvath D. S4MPLE-sampler for multiple protein-ligand entities: methodology and rigid-site docking benchmarking. Molecules 20(5), 8997-9028 (2015).

46. Tanchuk VY, Tanin VO, Vovk AI, Poda G. A new scoring function for molecular docking based on autodock and autodock vina. Curr. Drug Discov. Technol. 12(3), 170-178 (2015).

47. Tanchuk VY, Tanin VO, Vovk AI, Poda G. A new, improved hybrid scoring function for molecular docking and scoring based on autodock and autodock vina. Chem. Biol. Drug Des. 87(4), 618-625 (2016).

48. Brylinski M. Nonlinear scoring functions for similarity-based ligand docking and binding affinity prediction. J. Chem. Inf. Model. 53(11), 3097-3112 (2013).

49. Kadukova M, Grudinin S. Docking of small molecules to farnesoid X receptors using autodock vina with the convex-PL potential: lessons learned from D3R Grand Challenge 2. J. Comput. Aided Mol. Des. 32(1), 151-162 (2018).

50. Athanasiou C, Vasilakaki S. Using physics-based pose predictions and free energy perturbation calculations to predict binding poses and relative binding affinities for FXR ligands in the D3R Grand Challenge 2. J. Comput. Aided Mol. Des. 32(1), 21-44 (2018).

51. Cole JC, Murray CW, Nissink JW, Taylor RD, Taylor R. Comparing protein-ligand docking programs is difficult. Proteins 60(3), 325-332 (2005).

-. The important ideas about root-mean-square deviation (RMSD).

52. Schnur DM, Grieshaber MV, Bowen JP. Development of an internal searching algorithm for parameterization of the MM2/MM3 force fields. J. Comput. Chem. 12(7), 844-849 (1991).

53. Halgren TA. Merck molecular force field. I. Basis, form, scope, parameterization, and performance of MMFF94. J. Comput. Chem. 17(5-6), 490-519 (1996).

54. Mayo SL, Olafson BD, Goddard WA. DREIDING: a generic force field for molecular simulations. J. Phys. Chem. 94(26), 8897-8909 (1990).

55. Peng Z, Ewig CS, Hwang M-J, Waldman M, Hagler AT. Derivation of Class II Force Fields. 4. van der Waals parameters of alkali metal cations and halide anions. J. Phys. Chem. A 101(39), 7243-7252 (1997).

56. Ewig CS, Berry R, Dinur U et al. Derivation of class II force fields. VIII. Derivation of a general quantum mechanical force field for organic compounds. J. Comput. Chem. 22(15), 1782-1800 (2001).

57. Maple JR, Dinur U, Hagler AT. Derivation of force fields for molecular mechanics and dynamics from ab initio energy surfaces. Proc. Natl Acad. Sci. USA 85(15), 5350-5354 (1988).

58. Dinur U, Hagler Arnold T. New approaches to empirical force fields In: Lipkowitz KB, Boyd DB. (Eds). Reviews in Computational Chemistry. Wiley-VCH, Inc. (2007).

59. Verkhivker GM, Bouzida D, Gehlhaar DK et al. Deciphering common failures in molecular docking of ligand-protein complexes. J. Comput. Aided Mol. Des. 14(8), 731-751 (2000). 
60. Gehlhaar DK, Verkhivker GM, Rejto PA et al. Molecular recognition of the inhibitor AG-1343 by HIV-1 protease: conformationally flexible docking by evolutionary programming. Chem. Biol. 2(5), 317-324 (1995).

61. Lin YT, Huang KJ, Tseng CK, Chen KJ, Wang HM, Lee JC. Efficient in silico assay of inhibitors of hepatitis C Virus RNA-dependent RNA polymerase by structure-based virtual screening and in vitro evaluation. Assay Drug Dev. Technol. 9(3), 290-298 (2011).

62. Brooks BR, Bruccoleri RE, Olafson BD, States DJ, Swaminathan S, Karplus M. CHARMM: a program for macromolecular energy, minimization and dynamics calculations. J. Comput. Chem. 4(2), 187-217 (1983).

63. Vasudevan SR, Singh N, Churchill GC. Scaffold hopping with virtual screening from $\mathrm{IP}_{3}$ to a drug-like partial agonist of the inositol trisphosphate receptor. Chembiochem 15(18), 2774-2782 (2014).

64. Schneider G, Neidhart W, Giller T, Schmid G. 'Scaffold-hopping' by topological pharmacophore search: a contribution to virtual screening. Angew. Chem. Int. Ed. Engl. 38(19), 2894-2896 (1999).

65. Varma H, Lo DC, Stockwell BR. Frontiers in neuroscience high-throughput and high-content screening for huntington's disease therapeutics. In: Lo DC, Hughes RE. (Eds). Neurobiology of Huntington's Disease: Applications to Drug Discovery. CRC Press/Taylor \& Francis, FL, USA (2011).

66. Yang Z, Tam KY. Anti-cancer synergy of dichloroacetate and EGFR tyrosine kinase inhibitors in NSCLC cell lines. Eur. J. Pharmacol. 789, 458-467 (2016)

67. Xu R, Shen H, Guo R, Sun J, Gao W, Shu Y. Combine therapy of gefitinib and fulvestrant enhances antitumor effects on NSCLC cell lines with acquired resistance to gefitinib. Biomed. Pharmacother. 66(5), 384-389 (2012).

68. Wang Z, Wu X, Wang $\mathrm{L}$ et al. Facile and efficient synthesis and biological evaluation of 4-anilinoquinazoline derivatives as EGFR inhibitors. Bioorg. Med. Chem. Lett. 26(11), 2589-2593 (2016).

69. Kharbanda A, Runkle K, Wang W, Witze ES. Induced sensitivity to EGFR inhibitors is mediated by palmitoylated cysteine 1025 of EGFR and requires oncogenic Kras. Biochem. Biophys. Res. Commun. 493(1), 213-219 (2017).

70. Meng F, Wang F, Wang L, Wong SC, Cho WC, Chan LW. MiR-30a-5p overexpression may overcome EGFR-inhibitor resistance through regulating PI3K/AKT signaling pathway in non-small cell lung cancer cell lines. Front. Genet. 7, 197 (2016).

71. Chen L, Zhang Y, Liu J et al. Novel 4-arylaminoquinazoline derivatives with (E)-propen-1-yl moiety as potent EGFR inhibitors with enhanced antiproliferative activities against tumor cells. Eur. J. Med. Chem. 138, 689-697 (2017). 